\title{
Tsc1 haploinsufficiency leads to Pax2 dysregulation in the developing murine cerebellum
}

Ines Serra ${ }^{1, \#}$, Ana Stravs ${ }^{1,2 \#}$, Catarina Osório ${ }^{1}$, Maria Roa Oyaga $^{1}$, Martijn Schonewille $^{1}$, Christian Tudorache ${ }^{2}$, Aleksandra Badura ${ }^{1}$

${ }^{1}$ Department of Neuroscience, Erasmus MC, Rotterdam, 3015 CN, The Netherlands

${ }^{2}$ Institute of Biology Leiden, Leiden University, Leiden, 2333 BE, The Netherlands

\# These authors contributed equally to this work.

\section{Correspondence:}

Aleksandra Badura (‥badura@erasmusmc.nl)

Department of Neuroscience, Erasmus MC

Wytemaweg 80, 3015 CN Rotterdam

tel: 0031-(0)10 7043589 


\section{Abstract}

2 Tuberous sclerosis complex 1 (TSC1) is a tumour suppressor gene that inhibits the mechanistic

3 target of rapamycin (mTOR) pathway. Mutations in TSC1 lead to a rare complex disorder of

4 the same name, in which up to $50 \%$ of patients present with autism spectrum disorder (ASD).

5 ASD is a highly prevalent, early-onset neurodevelopmental disorder, characterized by social

6 deficits and repetitive behaviours, although the type and severity of symptoms show wide

7 variability across individuals. Amongst different brain areas proposed to play a role in the

8 development of ASD, the cerebellum is commonly reported to be altered, and cerebellar-

9 specific deletion of $T s c 1$ in mice is sufficient to induce an ASD-like phenotype. Given that the

10 mTOR pathway is crucial for proper cell replication and migration, this suggests that dysregulation of this pathway, particularly during critical phases of cerebellar development, could contribute to the establishment of ASD.

Here, we used a mouse model of TSC to investigate gene and protein expression during embryonic and early postnatal periods of cerebellar development. We found that, at E18 and P7, mRNA levels of the cerebellar inhibitory interneuron marker Pax2 were dysregulated. This was accompanied by changes in the expression of mTOR pathway-related genes and downstream phosphorylation of S6. Differential gene correlation analysis revealed dynamic changes in correlated gene pairs across development, with an overall loss of correlation between mTOR- and cerebellar-related genes in Tsc1 mutants compared to controls. We corroborated the genetic findings by characterizing the mTOR pathway and cerebellar development on protein and cellular levels with Western blot and immunohistochemistry. We found that Pax2-expressing cells were hypertrophic at E18 while, at P7, their number was increased and maturation into parvalbumin-expressing cells delayed. Our findings indicate that E18 and P7 are crucial time points in cerebellar development in mice that are particularly susceptible to mTOR pathway dysregulation. 


\section{Manuscript contribution to the field}

27 ASD is one of the most prevalent neurodevelopmental disorders, however little is known about the shared mechanisms underlying its aetiology. At the anatomical level, the cerebellum has been identified as one of the key structures involved in the development of ASD, whereas at the molecular level, mutations in the mTOR signalling pathway, essential for cell growth and proliferation, carry a high genetic risk for this disorder. We used a haploinsufficient tuberous sclerosis complex 1 (Tscl) mouse model to investigate the effects of mTOR overactivation in the developing cerebellum. Tscl inhibits the mTOR pathway, and mice with cerebellar-specific deletion of $T s c 1$ have been shown to harbour an ASD-like phenotype. We found that Pax2 expression in the cerebellum is dysregulated at prenatal and early postnatal time points, leading to a delayed maturation of inhibitory interneurons. Our findings indicate that mTOR overactivity in the cerebellum selectively affects the development of cerebellar interneurons. This finding is in line with other studies, which found decreased numbers of inhibitory interneurons in other models of ASD. Therefore, deficits in the maturation of the inhibitory signalling could be one of the mechanisms integrating high-risk mutations that underlie ASD aetiology. 


\section{Introduction}

54 The mechanistic target of rapamycin (mTOR) pathway is a highly complex, conserved and ubiquitous signalling avenue involved in biomass synthesis, growth and cell proliferation (1). Specifically during brain development, it has been proposed that a tight regulation of mTOR signalling is required for sustaining cell cycle length and re-entry, defining pluripotency status and triggering differentiation (2-4). However, how the mTOR pathway affects distinct lineages of differentiating cells is still largely unexplored. Supporting a crucial role for the mTOR signalling in brain development, mutations along this pathway frequently lead to complex monogenic neurodevelopmental disorders (also known as mTORopathies), characterized by heterogeneous neuropsychiatric phenotypes that include megalencephaly, epilepsy, intellectual disability and autism spectrum disorder (ASD) $(5,6)$. dominant disorder affecting 1 in 6000 people, that arises from heterozygous mutations in the TSC1 or TSC2 genes $(7,8)$. As TSC1 and 2, together with TBC1D7, form a tumour suppressor complex upstream of mTOR, loss of function of this complex leads to mTOR pathway overactivity $(9,10)$. mTOR can be organized in two complexes, mTORC1 and mTORC2, characterized, among others, by the presence of Raptor and Rictor, respectively $(11,12)$. While mTORC1 is primarily associated with growth and proliferation, mTORC2 regulates cytoskeleton organization and cell motility $(13,14)$. Nonetheless, crosstalk between mTORC1 and mTORC2 is vast, and changes in the function of both complexes due to mTOR dysfunction were shown to alter dendritic arbour morphology and synaptic transmission (15). On the whole, the effects of mTOR overactivity in TSC patients lead to a multi-system phenotype that includes widespread hamartoma growth, high prevalence of epilepsy, and, in up to $50 \%$ of the patients, ASD $(16,17)$. 
ASD is characterized by deficits in social communication and interaction, and by the presence of restricted, repetitive, and inflexible behaviours (18). The World Health Organization (WHO) estimates that 1 in 160 children worldwide will present with ASD, although its prevalence is known to vary across nations (19). Despite this high prevalence, little is known about the molecular mechanisms that underlie ASD. This is due to a significant knowledge gap, particularly with respect to brain development, when even limited signalling alterations can translate into considerable brain function, connectivity and structural deficits (20-22). While there is no single major anatomical abnormality evident in all people with ASD, the cerebellum is a brain structure that has emerged as a significant putative contributor to the development of ASD phenotypes. In humans, damage to the cerebellum is the second largest factor contributing to the risk of developing ASD (23-25), while cerebello-cortical connectivity is often found to be impaired in people with $\operatorname{ASD}(26,27)$. In recent years, several studies showed that murine models with cerebellar-specific deletion or inactivation of genes affecting the mTOR pathway, replicate these human phenotypes, presenting with decreased social interaction, increased repetitive behaviours and inflexible learning (28-31). Together with the fact that many mTOR pathway genes are found to be enriched in the cerebellum $(32,33)$, this suggests that this brain area may be particularly sensitive to changes in mTOR pathway regulation.

Here, we used a haploinsufficient $T s c 1$ mouse model, mimicking the human genotype of TSC, to investigate the effects of TSC1 deficiency in the developing cerebellum. We found that genetic dysregulation of the mTOR pathway can be detected from E18, suggesting a compensatory down-regulation in response to the hyperactivity of this pathway. Changes to cerebellar development can also be found at this age and postnatally at P7. Specifically, we found that Pax 2 expression at these time points is altered, indicative of a delay in its expression in $\mathrm{Tscl}^{+/}$mice. This culminated in slowed maturation and reduced parvalbumin expression. 
102 Overall, our data suggest that mTOR overactivity in the cerebellum preferentially affects the

103 development of cerebellar interneurons, which could potentially promote the development of

104 altered circuitry and, consequently, lead to behavioural deficits.

105

106

107

108

109

110

111

112

113

114

115

116

117

118

119

120

121

122

123

124

125

126 


\section{Materials and Methods}

\section{Mouse procedures}

Timed pregnancies were established between wild-type C57BL/6 females $\left(\right.$ Tsc $\left.^{+/+}\right)($Charles River Laboratories) and $T s c 1^{\mathrm{tm} 1 \mathrm{Djk}}\left(T s c 1^{+/-}\right)$males to obtain mixed $T s c 1^{+/+}$and $T s c 1^{+/-}$litters (34). Vaginal plugs were checked daily, and embryonic day 0 (E0) was defined when a plug was observed. Confirmed pregnant dams were individually housed. Mice were maintained on a standard $12 \mathrm{~h}$ light/dark cycle, with access to food and water ad libitum. to cervical dislocation, and E15 ( $\mathrm{n}=8$ mice per genotype) and E18 ( $=6$ mice per genotype) embryos collected onto cold PBS on ice. For the collection of neonatal samples, P1 ( $n=8$ mice per genotype) and P7 ( $\mathrm{n}=8$ mice per genotype) mice were anesthetized prior to decapitation. or dry ice, respectively, and kept at $-80^{\circ} \mathrm{C}$ until used.

All experimental animal procedures were approved a priori by an independent animal ethical committee (DEC-Consult, Soest, The Netherlands), as required by Dutch law, and conform to the relevant institutional regulations of the Erasmus MC and Dutch legislation on animal experimentation.

\section{Real-time qPCR}

\section{Primer design}

148 Seven genes of interest along the TSC-mTOR pathway (Tsc1, Tsc2, Rictor, Rptor, Mtor, 149 Rps6kb1 and Rps6) (1) and 5 genes representing distinct cerebellar lineages (Pax2, Pax6, 150 Calb1, Slcla3 and Gdf10) (35) were targeted. Housekeeping genes were selected based on 151 previous literature using embryonic and neonatal mouse brain tissue (36-38). Two 
bioRxiv preprint doi: https://doi.org/10.1101/2021.12.08.471768; this version posted December 8, 2021. The copyright holder for this preprint (which was not certified by peer review) is the author/funder, who has granted bioRxiv a license to display the preprint in perpetuity. It is made available under aCC-BY-NC-ND 4.0 International license.

152 housekeeping genes were selected per age: Ywhaz and Sdha were used for the E15 group, Gusb 153 and Sdha for E18, and Gusb and Ywhaz for P1 and P7.

Primer pairs were adapted from literature or designed using Primer-BLAST

155 (ncbi.nlm.nih.gov/tools/primer-blast) and Ensembl (m.ensembl.org) (Table 1). 


\begin{tabular}{|c|c|c|}
\hline Targeted gene & Primer: Forward sequence & Primer: Reverse sequence \\
\hline \multicolumn{3}{|c|}{ qPCR } \\
\hline Calb1 & TCTGGCTTCATTTCGACGCTG & ACAAAGGATTTCATTTCCGGTGA \\
\hline Gdf10 & CAGGACATGGTCGCTATCCAC & ACAGGCTTTTGGTCGATCATTTC \\
\hline Gusb & CACACTGACCCCTCATACCC & TGCAGTCCCGCATAGTTGAA \\
\hline Mtor & CACCAGAATTGGCAGATTTGC & CTTGGACGCCATTTCCATGAC \\
\hline $\operatorname{Pax} 2$ & AAGCCCGGAGTGATTGGTG & CAGGCGAACATAGTCGGGTT \\
\hline Pax6 & TACCAGTGTCTACCAGCCAAT & TGCACGAGTATGAGGAGGTCT \\
\hline Rptor & CAGTCGCCTCTTATGGGACTC & GGAGCCTTCGATTTTCTCACA \\
\hline Rictor & ACAGTTGGAAAAGTGGCACAA & GCGACGAACGTAGTTATCACCA \\
\hline Rps6kbl & AGCCCTGATGACTCCACTCT & CTGACAGGTGTTCGTGGACT \\
\hline Rsp6 & CTGGGTTAAGCGGAAGTCGG & CCACCTCGATGAGCTTCTGA \\
\hline Sdha & GGAACACTCCAAAAACAGACCT & CCACCACTGGGTATTGAGTAGAA \\
\hline Slcla3 & CCGACCGTATAAAATGAGCTACC & ATTCCTGTGACGAGACTGGAG \\
\hline$T s c 1$ & CGGCTCTGGAGGAACACAAT & GCTGACTGTATCGGGCTTGT \\
\hline$T s c 2$ & AGTTCTCACCTTATTGAAGGCCA & CATTGGAGGGGTAGTCCTTGA \\
\hline Ywhaz & GAAAAGTTCTTGATCCCCAATGC & TGTGACTGGTCCACAATTCCTT \\
\hline \multicolumn{3}{|c|}{ Genotyping } \\
\hline Tscl & $\begin{array}{l}\text { GTCACGACCGTAGGAGAAGC } \\
\text { AGGAGGCCTCTTCTGCTACC }\end{array}$ & GAATCAACCCCACAGAGCAT \\
\hline
\end{tabular}


(blast.ncbi.nlm.nih.gov), and in vitro with conventional PCR and melt curve analysis.

RNA extraction

161 Following cerebellar dissection, RNA was isolated using a standard chloroform/isopropanol method (39). In brief, tissue in TRI Reagent ${ }^{\circledR}$ (T9424, Sigma) was thawed, homogenized by syringe aspiration (G23 and G25) and vortexed. Chloroform (1:5) was added to the sample, followed by a 5-minute incubation at room temperature (RT). Samples were then centrifuged at $10800 \mathrm{~g}$, at $4^{\circ} \mathrm{C}$ for $15 \mathrm{~min}$. The aqueous phase was collected and a 1:1 ratio of isopropanol was added. Samples were centrifuged for $10 \mathrm{~min}$ at maximum speed ( 20 $000 \mathrm{~g})$. The obtained RNA pellet was washed twice with $70 \%$ ethanol, air-dried, resuspended in $20 \mu \mathrm{L}$ of RNasefree water (UltraPure ${ }^{\mathrm{TM}}$ DNase/RNase-Free Distilled Water, 10977-035, Invitrogen), and quantified with NanoDrop (Thermo Scientific).

$R T-q P C R$

RNA was transcribed using qScript ${ }^{\circledR}$ cDNA SuperMix (Quantabio, 95048-100), according to manufacturer's instructions. RT-qPCR was performed with PerfeCTa $^{\circledR}$ SYBR $^{\circledR}$ Green

174 FastMix $^{\circledR}$ (Quantabio, 95072-05K) following manufacturer's instructions, with $10 \mu \mathrm{M}$ of 175 forward and reverse primers, and $1 \mu \mathrm{L}$ cDNA (diluted 1:5). All samples were processed in 176 duplicates. RT-qPCR was performed in a CFX96 ${ }^{\mathrm{TM}}$ Real-Time PCR detection system (Bio$177 \mathrm{Rad}$ ), with initial denaturation for $1 \mathrm{~min}$ at $95^{\circ} \mathrm{C}$, followed by 40 cycles of $5 \mathrm{~s}$ at $95^{\circ} \mathrm{C}$, and 15 $\mathrm{s}$ at $55^{\circ} \mathrm{C}$, with melting curve generation. 
Raw data processing

Relative quantification was performed as in (40), on 8 biological samples per genotype for the cycle $(\mathrm{Cq})$ values were extracted for each sample (genes of interest and housekeeping genes), and the mean $\mathrm{Cq}$ per gene was calculated within the control group (WT). A $\Delta \mathrm{Cq}$ was then calculated per sample by subtracting the control group average from the sample mean Cq. For each sample, the relative quantities were then calculated $\left((1+\mathrm{E})^{\Delta \mathrm{Cq}}, \mathrm{E}=1\right)$. Normalized expression per sample (genes of interest) was obtained by dividing the relative quantity of a given sample by the geometric mean of the relative quantities of the two housekeeping genes. The average normalized expression of the samples, in each genotype per gene of interest, was calculated.

\section{Western blot}

194 Cerebellar tissue from E18 ( $\mathrm{n}=3$ mice per genotype) and P7 ( $\mathrm{n}=5$ mice per genotype), WT and ${T S c 1^{+/+}}$mice, was dissected and immediately frozen in dry ice. Samples were homogenized with a Dounce homogenizer in ice-cold lysis buffer containing $50 \mathrm{mM}$ Tris- $\mathrm{HCl} \mathrm{pH} 8,150 \mathrm{mM}$

$\mathrm{NaCl}, 1 \%$ Triton $\mathrm{X}-100,0.5 \%$ sodium deoxycholate, $0.1 \%$ SDS and protease inhibitor cocktail

(Roche). Protein concentrations were measured using a Pierce BCA protein assay kit (Thermo

Fisher). Samples were denatured and proteins separated in SDS-PAGE in Criterion ${ }^{\mathrm{TM}}$ TGX 200 Stain-Free ${ }^{\mathrm{TM}}$ Gels (Bio-Rad), and transferred onto nitrocellulose membranes with the TransBlot ${ }^{\circledR}$ Turbo ${ }^{\mathrm{TM}}$ Blotting System (Bio-Rad).

Membranes were blocked with 5\% BSA (Sigma-Aldrich) in Tris-buffered saline

204 with the following primary antibodies: Pax2 (1:1000, rabbit, Cell Signaling 9666), Phospho205 S6 Ribosomal Protein (Ser235/236) (1:1000, rabbit, Cell Signaling 2211), Ribosomal Protein 
S6 (1:1000, mouse, Santa Cruz SC-74459) or GAPDH (1:1000, mouse, Cell Signaling 97166).

Secondary antibodies used were goat anti-Rabbit Immunoglobulins/HRP (1:10000, Agilent

208

Dako P0448) or goat anti-Mouse Immunoglobulins/HRP (1:10000, Agilent Dako P0447).

Proteins were detected by the luminol-based enhanced chemiluminescence method

210 (SuperSignal $^{\mathrm{TM}}$ West Femto Maximum Sensitivity Substrate or SuperSignal ${ }^{\mathrm{TM}}$ West Dura

211 Extended Duration Substrate, Thermo Fisher). Membranes were stripped with Restore ${ }^{\mathrm{TM}}$ PLUS

212 Western Blot Stripping Buffer (Thermo Fisher). Densitometry of protein bands of interest was

213 normalised to that of GAPDH using the Image Studio Lite software (LI-COR Biosciences).

214

215 Immunohistochemistry

216 After collection, E18 embryos ( $\mathrm{n}=3$ mice per genotype) were fixed by immersion in cold $4 \%$

217 paraformaldehyde (PFA) in phosphate buffered saline (PBS). P7 pups ( $\mathrm{n}=3$ per genotype)

218 were injected with an overdose of pentobarbital and transcardially perfused with $4 \%$ PFA in

219 PBS. Afterwards, tissue was placed in 4\% PFA for 2 hours and transferred into $30 \%$ sucrose

220 in $0.1 \mathrm{M}$ phosphate buffer (PB) until embedding. Samples were embedded in $14 \%$ gelatine /

221

222

223

224

225

226

227

228

229

230

$30 \%$ sucrose and incubated in a 10\% PFA / $30 \%$ sucrose solution for $1.5 \mathrm{~h}$, at RT, on a shaker.

Embedded samples were kept in $30 \%$ sucrose $/ 0.1 \mathrm{M}$ PB at $4^{\circ} \mathrm{C}$ until cut.

Cerebellar samples were cut in $30 \mu \mathrm{m}$ sagittal sections using a cryomicrotome (Leica

SM 200R). Free-floating sections were rinsed with PBS and preincubated with $10 \%$ normal horse serum (NHS) / 0.5\% Triton ${ }^{\mathrm{TM}} \mathrm{X}-100$ in PBS, for $1 \mathrm{~h}$ at RT on a shaker. Sections were then incubated overnight, at $4^{\circ} \mathrm{C}$, in $2 \%$ NHS / $0.4 \%$ Triton ${ }^{\mathrm{TM}} \mathrm{X}-100$ in PBS with primary antibodies against Pax2 (1:500, rabbit, Invitrogen 71-6000), Calbindin D28-K (1:10.000, mouse, Sigma C9848) or Parvalbumin (1:500, mouse, Swant 235). The following day, sections were rinsed with PBS and incubated with AlexaFluor 594 (1:500, Donkey anti-rabbit, Jackson 711-585-152) and AlexaFluor 488 (1:500, Donkey anti-mouse, Jackson 715-545-150) in 2\% 
NHS / 0.4\% Triton ${ }^{\mathrm{TM}} \mathrm{X}-100$ in PBS, for $1.5 \mathrm{~h}$ at RT. Sections were rinsed, counterstained with

DAPI (1:10.000), and rinsed again with PB before mounting. Sections were imaged with a 10X

(E18) or 20X (P7) objective using a Zeiss AxioImager.M2 microscope.

\section{Microscopy images quantification}

The number and area of $\mathrm{Pax}^{+}$positive cells was automatically counted with Fiji ImageJ

(41), using custom-written macros (https://github.com/BaduraLab). Given the positive

for cell area (42). To calculate the distance between Pax $2^{+}$particles, we used the ND ImageJ plugin (43). around the visible cell body cross-section.

At P7, parvalbumin (PV) staining was still sparse and dispersed in the developing cerebellum. Thus, we opted for the measurement of the total area occupied by PV stain rather than counting individual cells. To do this, PV-stained cerebellar sections were automatically excluding the already existing Purkinje cell layer (see Results). This enabled the measurement of the PV-signal primarily derived from developing molecular layer interneurons (44).

Statistics

All statistical analysis was performed on GraphPad Prism 8. Data were first screened

254 for the presence of outliers using the ROUT method, and tested for normality using the

255 Shapiro-Wilk test, when applicable. When the normality assumption was followed, a two- 
256 tailed t-test was used for data comparison. When this assumption was violated, a two-tailed

257 Mann-Whitney test was used. Variable correlation was performed using Pearson's correlation,

258 and simple linear regression was used for line fitting.

259

260

261

262

263

264

265

266

267

268

269

270

271

272

273

274

275

276

277

278

279

280 
mTOR pathway and cerebellar cell type-specific gene transcription is dysregulated in

\section{$\operatorname{Tsc1}^{+/-}$cerebella}

To first investigate whether Tscl haploinsufficiency changes the expression of mTOR pathway and cerebellar genes in the developing cerebellum, we performed RT-qPCR in distinct developmental periods (36). Thus, based on available literature and inter-plate stability, we selected different housekeeping gene pairs for each time point analysed: Sdha and Ywhaz for E15, Gusb and Sdha for E18, and Gusb and Ywhaz for P1 and P7. Within each developmental time point, relative gene expression across the two genotypes, WT and $T s c 1^{+/}$, was compared.

Regarding the transcription of mTOR pathway genes, we found no differences between genotypes at E15, except for the expected down-regulation of $T s c l$ transcription $(t(13)=8.685$, $p<0.000001)$. At E18, in addition to $T s c 1(t(10)=9.320, p=0.000003)$, also the transcription of $\operatorname{Tsc} 2(t(10)=2.389, p=0.038)$, Rictor $(t(9)=2.521, p=0.033)$ and $\operatorname{Mtor}(t(10)=3.265$, $p=0.009)$ was down-regulated in $T s c 1^{+/-}$cerebella, suggesting the presence of down-regulation mechanisms regarding mTOR complex genes. At P1, Tscl $(t(14)=5.974, p=0.000034)$ and Rictor $(t(14)=2.171, p=0.048)$ were still down-regulated while, at P7, only Tsc1 $(t(14)=$ 3.873, $p=0.002)$ and $T s c 2(t(13)=2.218, p=0.045)$ were different from controls (Figure 1A).

We then analysed the transcription of genes involved in the specification of distinct cerebellar cell types. No difference between genotypes was detected at E15 nor at P1. However, at E18, we found that both $\operatorname{Pax} 2(t(10)=2.867, p=0.017)$, a marker for developing 
306 interneurons, and Slcla3 $(t(10)=2.561, p=0.028)$, a marker for Bergman glia, were down-

307 regulated in $T s c 1^{+/}$cerebella, while an up-regulation of $\operatorname{Pax} 2(t(13)=2.642, p=0.020)$ was

308 detected in P7 Tscl ${ }^{+-}$cerebellar samples (Figure 1B). These data indicate that, although the

309 mTOR pathway primarily undergoes post-translational regulation, during development, $\operatorname{Tsc}^{+/-}$

310 haploinsufficiency dysregulates a number of mTOR pathway-related genes, as well as the

311 relative expression of Slcla3 and Pax2. 
bioRxiv preprint doi: https:/doi.org/10.1101/2021.12.08.471768; this version posted December 8 , 2021. The copyright holder for this preprint (which was not certified by peer review) is the author/funder, who has granted bioRxiv a license to display the preprint in perpetuity. It is made available under aCC-BY-NC-ND 4.0 International license.

A

mTOR pathway genes
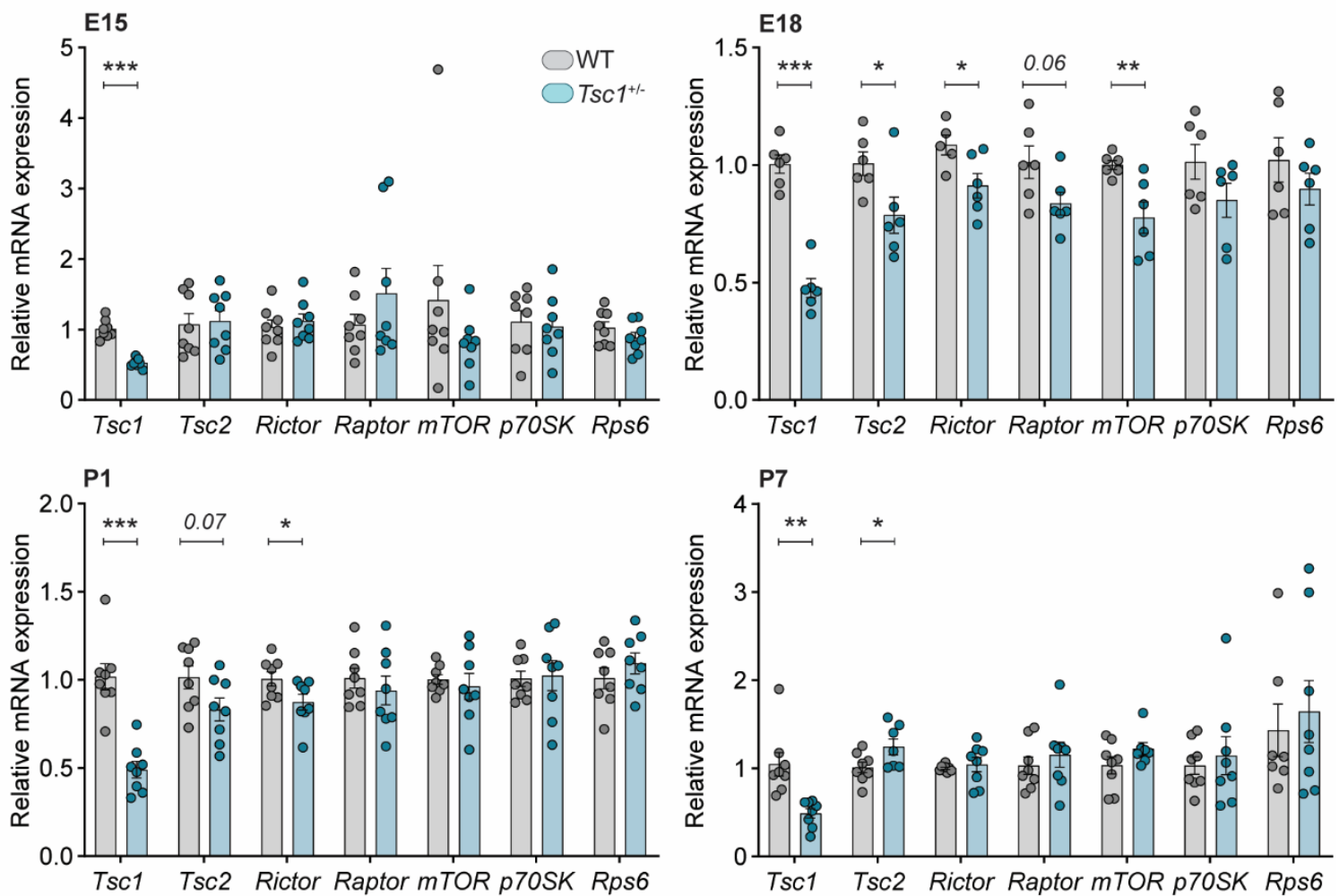

B

Cerebellar identity genes
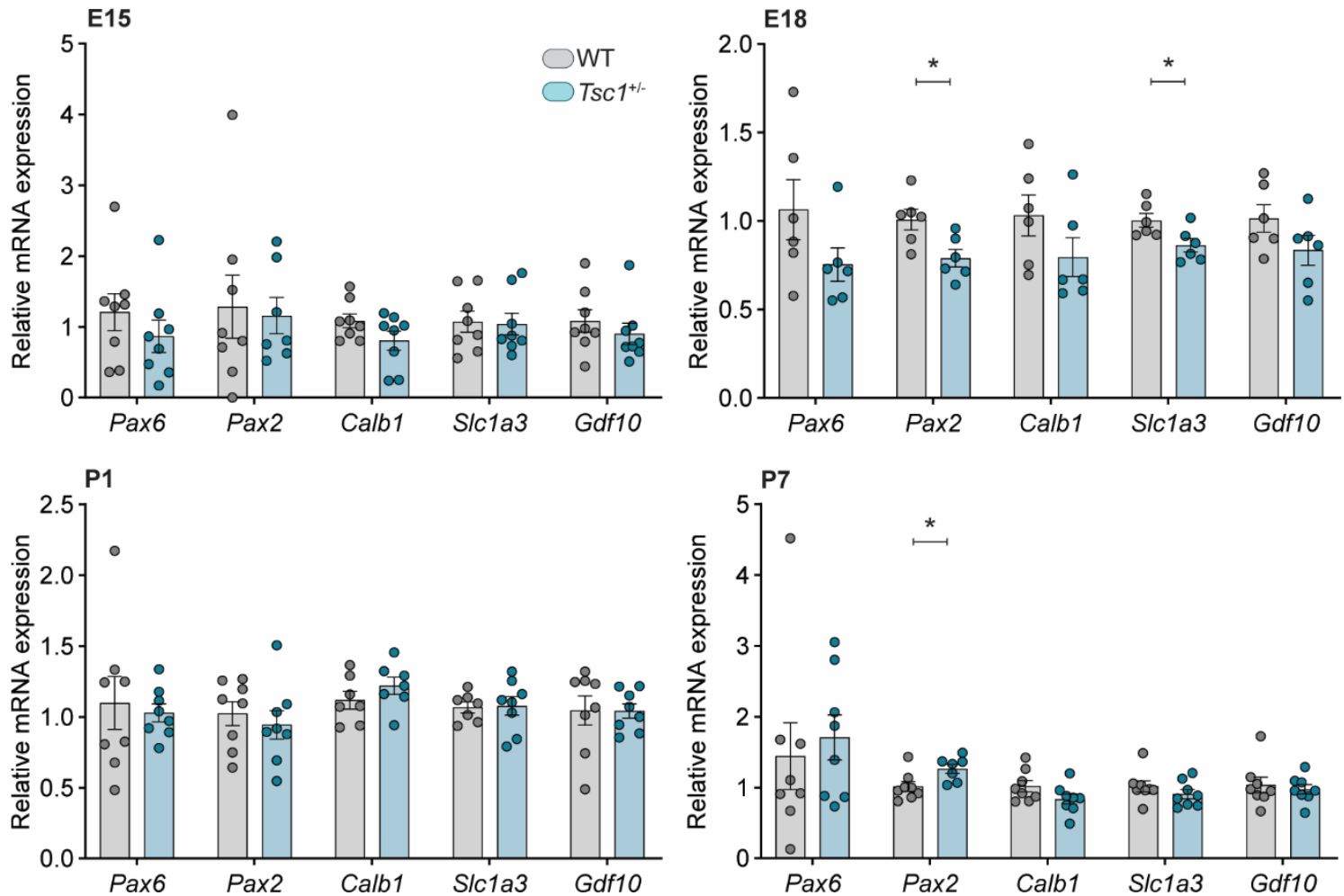

313 Figure 1: Relative gene expression is altered in $\boldsymbol{T s c 1 ^ { + / }}$ cerebella. mRNA expression of mTOR

314 pathway genes (A) and cerebellar cell type-specific genes (B), relative to housekeeping genes. (A) A 
switch in mTOR-related gene expression occurs at E18, a time point when most markers analysed were significantly down-regulated in $T s c 1^{+/}$cerebella. (B) Gene expression of cerebellar identity genes is relatively stable between genotypes, with the exception of Pax2 and Slc1a3. $t$-test, ${ }^{*} p<0.05,{ }^{* *} p<$ $0.01, * * * p<0.001 ; \mathrm{n}=8$ mice per genotype, except for $\mathrm{E} 18$, where $\mathrm{n}=6$.

319

\section{Tsc1 haploinsufficiency leads to dysregulated gene interactions}

Given that E18 and P7 presented with the largest differences between WT and $T s c 1^{+/}$ cerebellar gene expression, we focused on these two time points to evaluate the correlation between the relative expression of genes of interest. As expected, we found a significant positive correlation between the relative expression of $T s c 1$ and $T s c 2$ in WT cerebella (E18: $r^{2}$ $\left.=0.65, p=0.052 ; \mathrm{P7}: r^{2}=0.50, p=0.050\right)$. While this correlation was still present in $T s c 1^{+/}$ mice at $\mathrm{E} 18\left(r^{2}=0.91, p=0.003\right)$, by $\mathrm{P} 7$ this relation was lost in mutants $\left(r^{2}=0.03, p=0.718\right)$

(Figure 2A). Additionally, while the relative expression of $T s c 1$ in WT was negatively correlated with the relative expression of $S 6\left(\mathrm{E} 18: r^{2}=0.83, p=0.011 ; \mathrm{P} 7: r^{2}=0.64, p=0.03\right)$, this was not the case for $T s c 1^{+-}$mice $\left(\mathrm{E} 18: r^{2}=0.01, p=0.82 ; \mathrm{P} 7: r^{2}=0.03, p=0.71\right)$ (Figure 2B). This indicates that genetic mTOR pathway dysregulation in $T s c 1^{+/}$cerebella can be found early in development, likely prior to detectable protein changes, and that these deficits exhibit time-dependent progression.

Pax2 expression is initiated in inhibitory interneuronal precursors during their last mitosis (45). Possibly reflecting the positive role of the mTOR pathway on neuronal differentiation (46), we found a negative correlation between $T s c 1$ and $P a x 2$ relative expression in E18 WT cerebella $\left(r^{2}=0.74, p=0.03\right)$. However, this correlation was absent in $T s c 1^{+/}$mice $\left(r^{2}=0.22, p=0.35\right)$ (Figure 2C). This suggests that mTOR pathway disruption through Tsc1 haploinsufficiency could lead to early dysfunction of $\operatorname{Pax2}^{+}$cell differentiation. 
A

E18

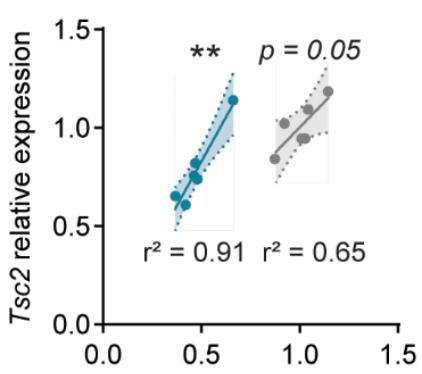

Tsc1 relative expression

B
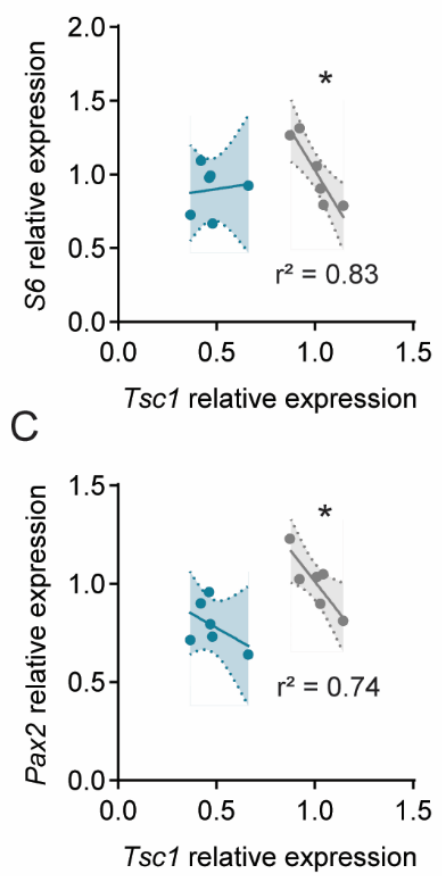

P7

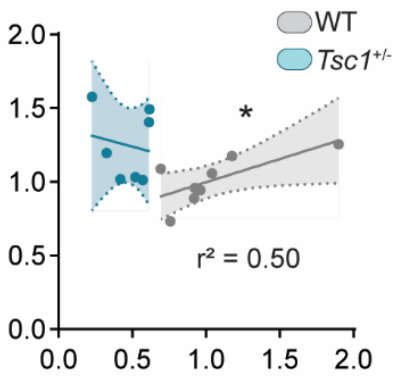

Tsc1 relative expression
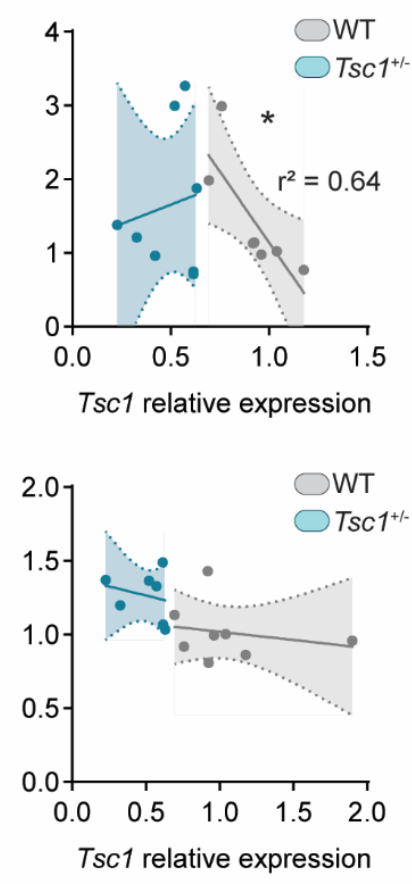

Figure 2: Gene correlation is lost in $\boldsymbol{T s c 1}^{+/}$cerebella. Pearson's correlation plots for mRNA relative expression of $T s c 1$ over (A) Tsc2, (B) $S 6$ and (C) Pax2 in E18 (left column) and P7 (right column) cerebella. Pearson's correlation, $* p<0.05, * * p<0.01 ; \mathrm{n}=8$ mice per genotype, except for E18, where $\mathrm{n}=6$.

Tsc1 haploinsufficiency increases the size of $\mathrm{Pax}^{+}$cells but does not affect cell number at E18 in Golgi cells, and continues into postnatal time points, with the differentiation of stellate and basket cells perinatally (47-49). Having found that the relative mRNA expression of Pax2 was 
down-regulated in $\mathrm{Tsc}^{+/-}$cerebella at E18, we then investigated the protein expression of Pax2

Correspondingly, we also found that WT and $\mathrm{Tscl}^{+/-}$cerebella presented with a similar number of Pax2-positive $\left(\operatorname{Pax} 2^{+}\right)$cells $(U=127.5, p=0.16)$ (Figure 3B, C).

At this stage, $\mathrm{Pax} 2^{+}$cells are highly migratory and quasi-uniformly dispersed through the developing cerebellum (45). We corroborated this uniform positioning in both WT and $T s c 1^{+/}$sections as, in the two genotypes, the total number of Pax $2^{+}$cells was positively correlated with the correspondent section size (WT: $r^{2}=0.44, p=0.005 ; T s c 1^{+/-}: r^{2}=0.36, p=$ 0.003) (Figure 3D). To investigate the position of these cells, we then calculated the nearest neighbour distance between Pax $2^{+}$cells, and used this measure as a proxy for cell migration. We found that both WT and $T s c 1^{+/} \mathrm{Pax}^{+}$cells were separated by similar distances $(t(36)=$ 0.26, $p=0.84$ ) (Figure 3E). This suggests that, while $T s c 1$ haploinsufficiency causes a reduction in $\operatorname{Pax} 2$ transcription, this is not sufficient to affect the generation nor migration of

$364 \mathrm{Pax}^{+}$cells at this stage in development. by changes in neuronal cell size (50). Thus, we then measured the size of Pax $2^{+}$cells in the E18 cerebellum. Despite not finding increased overall levels of pS6 (Ser235/236)/total S6 in whole cerebellar extracts of $T s c 1^{+/}$mice $(U=4, p>0.99)$ (Figure 3A), we found that these

369 mice presented with enlarged Pax $2^{+}$cells when compared to WT cells $(t(36)=3.38, p=0.002)$

370 (Figure 3F). These larger cells were present across the full spectrum of measured areas (Figure 371 3G). This data suggests that the mTOR pathway is overactive in $T s c 1^{+/-}$interneuronal 372 progenitor cells, leading to an overall increase in cell size that seems to affect distinct lineages 373 of inhibitory interneurons. 
A

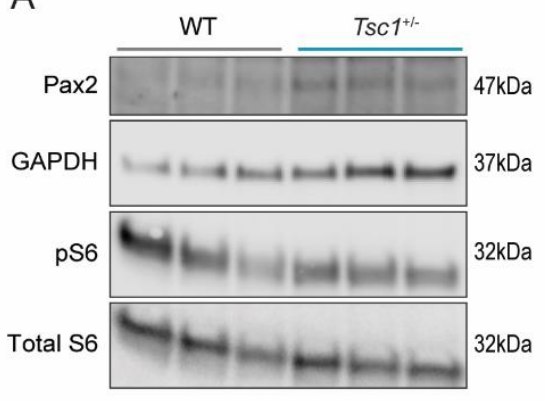

B

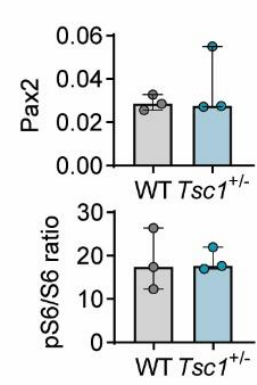

C
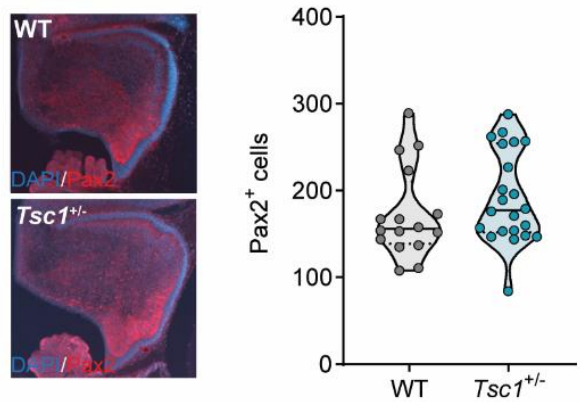

G

$\mathrm{F}$

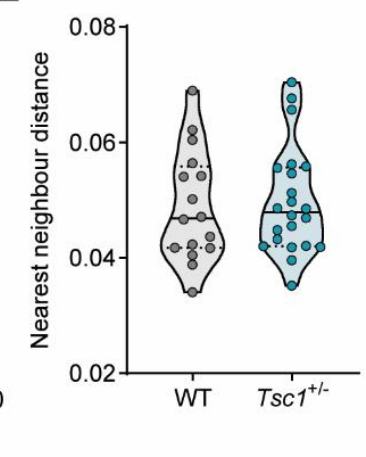

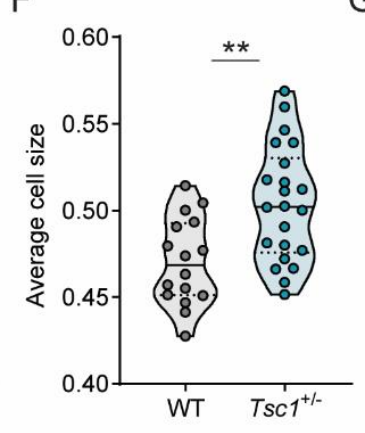

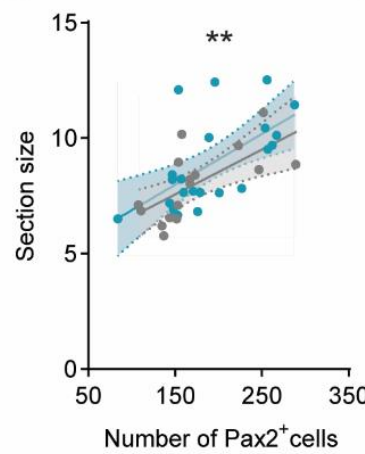

E

375 in inches. ${ }^{* *} p<0.01$.

\section{development in $\mathrm{Tsc1}^{+/-}$mice}

Figure 3: Increased Pax2 ${ }^{+}$cell size is found in $\mathbf{E} 18 \mathbf{T s c}^{+/-}$cerebella. (A) Western blot quantification of Pax2, phosphorylated S6 (Ser235/236) and total S6, in whole cerebellar tissue ( $\mathrm{n}=3$ mice per genotype, Mann-Whitney test). (B) Representative Pax2-stained sagittal section (red) counterstained with DAPI (blue). (C) Pax $2^{+}$cell count on WT ( $\mathrm{n}=16$ sections from 2 mice) and $T s c 1^{+-}(\mathrm{n}=22$ sections from 2 mice) sagittal sections (Mann-Whitney test). (D) Pearson's correlation between cerebellar section size and the number of Pax $2^{+}$cells (WT in grey, $T s c 1^{+/-}$in blue; 16 points for WT and 22 for $T s c 1^{+/}$mice). (E-F) Nearest neighbour distance and average cell size of WT and $T s c 1^{+/-} \mathrm{Pax}^{+}$cells (n $=16$ sections from 2 mice for WT and $\mathrm{n}=22$ sections from 2 mice for $T s c 1^{+/} ; t$-test). (G) Frequency of $\mathrm{Pax}^{+}$cells over the binned cell area (2863 cells from WT and 4252 cells from $T s c 1^{+/}$mice). Area

\section{Overactive mTOR pathway in $\mathrm{P7}$ cerebellum leads to perturbed interneuron}

At P7, although the vast majority of cerebellar interneurons is already generated, these can be found in distinct developmental stages, from migrating to fully mature neurons $(49,51)$. 
390 To further explore the increase in Pax2 relative expression we previously found in $T_{s c 1^{+/}}$ 391 cerebella at P7, we next analysed the expression and distribution of Pax $2^{+}$cells in the P7 cerebellum. As observed for the E18 cohort, we found no difference in Pax2 protein expression between WT and $T s c 1^{+/-}$cerebella $(t(8)=1.61, p=0.76)($ Figure 4A). Supporting our qPCR data, immunohistochemical analysis of Pax2-labelled cerebellar sections revealed that $T s c 1^{+/}$ cerebella presented with an increased number Pax2 ${ }^{+}$cells (Figure $4 \mathbf{B}, \mathbf{C}$ ). In addition, these cells were most abundant in the cerebellar white matter (Figure 4B), altogether suggesting the presence of an immature Pax2 phenotype in $T s c 1^{+/}$mice $(52,53)$. in $\mathrm{Tscl}^{+/}$mice compared to WT $(U=2329, p=0.08)$ (Figure 4D). This putative Pax $2^{+}$cell size change appeared to be specific towards $\mathrm{Pax} 2^{+}$cells, as we found no difference in the size of other GABAergic cells, such as Purkinje cells $(t(58)=0.1219, p=0.90)$ (Figure 4E, F).

404 Thus, we further investigated $\mathrm{Pax} 2^{+}$cell size by analysing their frequency distribution across 405 distinct binned areas. We found that $T s c 1^{+/-}$mice presented with an increased number of small $406 \mathrm{Pax}^{+}$cells (Figure 4G), which likely represent stellate and basket cells in the nascent 407 molecular layer (45). Contrarily, $T s c 1^{+/-}$mice exhibited decreased numbers of large Pax $2^{+}$cells, 408 indicative of the granular layer interneurons, Golgi cells $(44,54)$ (Figure 4H). Furthermore, 409 small $\mathrm{Pax}^{+}$cells in $T s c 1^{+/-}$mice were slightly smaller than WT cells $\left(\mathrm{WT}=132\right.$ vs $T s c 1^{+/}=$ 410123 , median size, $p<0.0001$ ) (Figure 4G-inset), as were large the Pax $2^{+}$cells (WT $=584$ vs $411 \mathrm{Tscl}^{+/-}=572$ median size, $p<0.0001$ ) (Figure 4H-inset). This suggests that Tscl 412 haploinsufficiency differentially alters Pax $2^{+}$-derived lineages. 
bioRxiv preprint doi: https://doi.org/10.1101/2021.12 08.471768; this version posted December 8,2021 . The copyright holder for this preprint (which was not certified by peer review) is the author/funder, who has granted bioRxiv a license to display the preprint in perpetuity. It is made available under aCC-BY-NC-ND 4.0 International license.

A

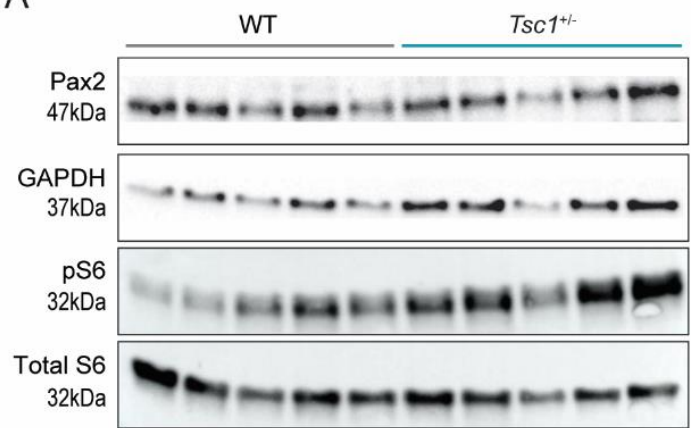

C

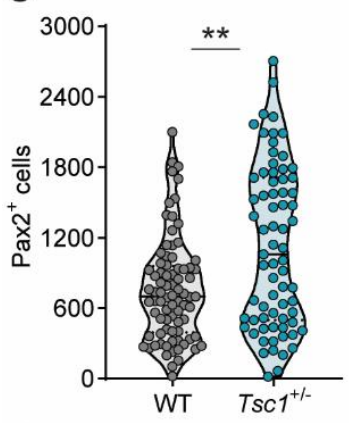

G

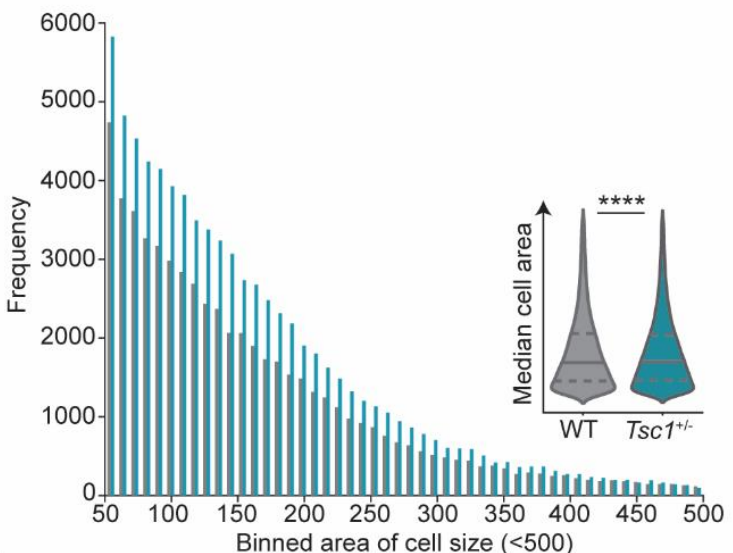

1

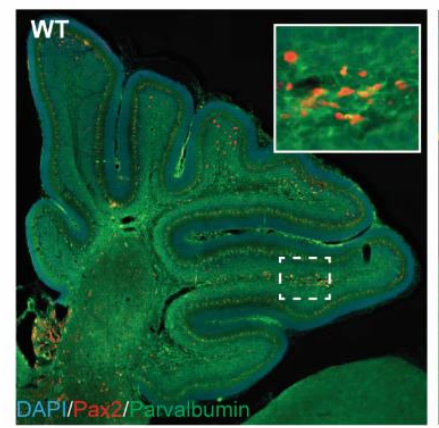

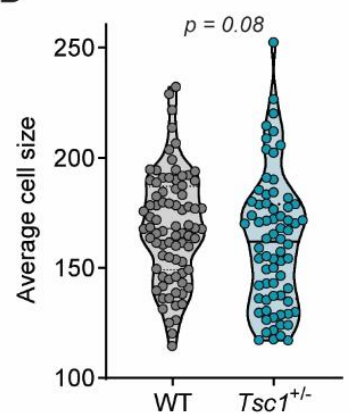

$\mathrm{H}$
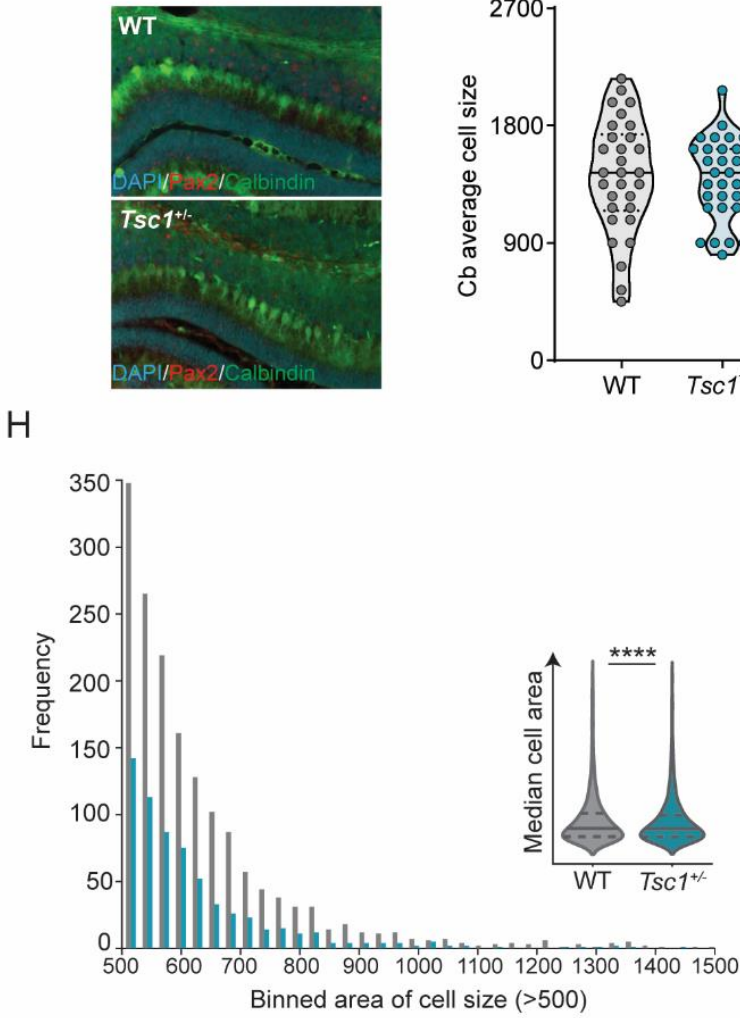

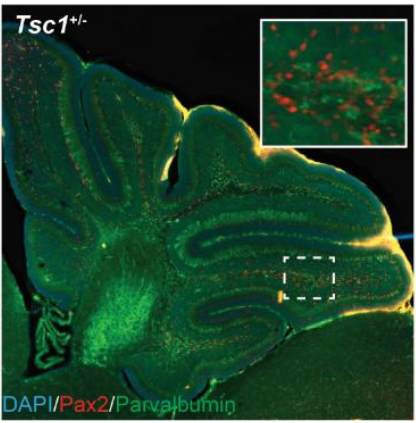

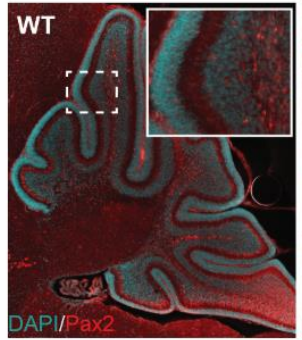

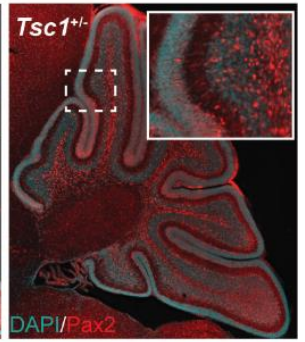

E

F

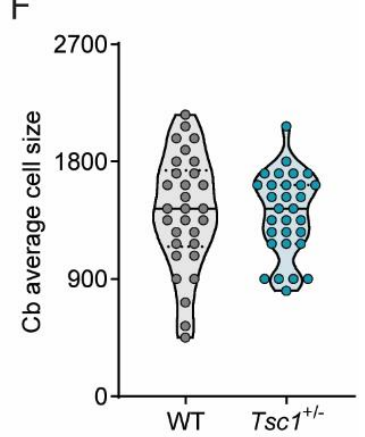

$\mathrm{J}$

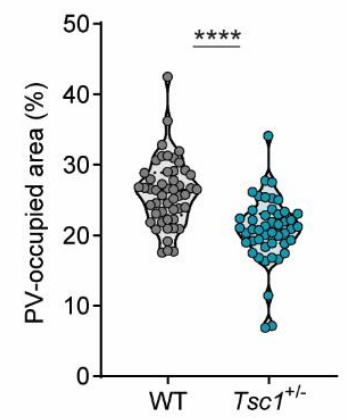

414 Figure 4: Tsc1 $^{+-}$mice present with interneuron development and maturation deficits at P7. (A)

415 Western blot quantification of Pax2, phosphorylated S6 (Ser235/236) and total S6, in whole cerebellar 416 tissue ( $\mathrm{n}=5$ mice per genotype; $t$-test). (B) Representative Pax2-stained sagittal section (red) 417 counterstained with DAPI (blue). (C) Pax $2^{+}$cell count on WT $\left(\mathrm{n}=81\right.$ sections from 3 mice) and $T s c 1^{+/}$ 
418 ( $\mathrm{n}=71$ sections from 3 mice) sagittal sections (Mann-Whitney test). (D) Average cell size of WT and

$419 T s c 1^{+/}$total Pax2 ${ }^{+}$cells $\left(\mathrm{n}=81\right.$ sections from 3 WT mice and $\mathrm{n}=71$ sections from $3 T_{s c 1^{+/}}$mice;

420 Mann-Whitney test). (E) Representative sagittal section stained for Calbindin (green), Pax2 (red) and

421 counterstained with DAPI (blue). (F) Average cell size of WT and $T s c 1^{+/}$Purkinje cells, measured

422 between lobules V and VI ( $\mathrm{n}=10$ cells per mouse, 3 mice per genotype; $t$-test). (G-H) Frequency of

423 small (left) and large (right) Pax2 $2^{+}$cells over binned cell area (61 189 cells from WT and 79955 cells

424 from $T s c 1^{+/}, 3$ mice per genotype). Insets show the median area of the measured cells. (I)

425 Representative section stained for PV (green), Pax2 (red) and DAPI (blue) (Mann-Whitney test). (J)

426 Percentage of PV-occupied area per section ( $n=51$ section from 3 WT mice and $n=49$ sections from

$4273 T_{s c 1}{ }^{+/}$mice; Mann-Whitney test). Area in pixels. $* p<0.05, * * p<0.01, * * * p<0.001$.

428

\section{Maturation of interneuron precursors at $\mathrm{P} 7$ is altered in $T s c 1^{+/-}$cerebella}

The density of cerebellar interneurons remains relatively constant between P5 to P10

431

(55). For the majority of these cells, comprising mostly of the molecular layer interneurons,

down-regulation of Pax 2 is accompanied by up-regulation of $\mathrm{PV}$, a process that marks interneuronal maturation $(44,56)$. Given that we found an increase in the number of Pax $2^{+}$cells down-regulation rather than an overall increase in cell generation. Therefore, we focused on the maturation of the interneuronal cells.

As a proxy of cell maturation, we quantified the percentage of cerebellar P7 area occupied by PV staining (see methods) (Figure 4I). We found that $T s c 1^{+/-}$cerebellar sections presented with a decreased percentage of surface area labelled by PV staining when compared to WT mice (median percentage: $26.45 \%$ for WT vs $20.89 \%$ for $T s c 1^{+/}, p<0.0001$ ), suggesting 441 that $T s c 1$ haploinsufficiency is accompanied by deficits in the maturation of molecular layer 442 interneurons (Figure 4J). 
Discussion

The mTOR pathway has been linked to many cellular and metabolic events, including

447 cell replication, growth and biomass production (1). Although the mTOR kinase is known to

448 be required for the formation of the central nervous system $(\mathrm{CNS})(2,4)$, its precise role in the

449 development of distinct cell lineages is not completely understood. Currently, a tight regulation

450 of the timing of mTOR pathway activation appears to be essential for the balance between

undifferentiated cell proliferation and cell differentiation. In postnatal mice, mTOR signalling

452 is detected in proliferating neural stem cells (NSCs), while knockdown of its activity reduces

453 proliferation. Conversely, increasing mTOR activation leads to a higher number of terminally

differentiated NSCs at the expense of their renewal (4). Further, in cortical interneuron

progenitors, deletion of mTOR decreases proliferation, leading to a reduction of mature

calbindin-positive cells (57).

In the developing cerebellum, few reports have addressed the role of the mTOR pathway in the specification of distinct cell types. Proper mTOR signalling appears imperative 459 for the correct development of Purkinje cells (PC), as disruptions in either mTORC1 or 460 mTORC2 signalling in PC from E17.5 lead to smaller soma size and deficits in dendritic arborization $(58,59)$. Conditional deletion of Rictor, essential for mTORC2 kinase activity,

462 from all CNS precursor cells at E10.5, induces early postnatal changes to PC, including the 463 emergence of several primary dendrites and abnormal vermal macrostructure (59).

464 Furthermore, while the loss of Rictor does not seem to affect the development of cerebellar 465 granule neuron precursor cells (GNP), deleting Raptor, necessary for mTORC1 activity, leads 466 to a decrease in cell number (60). Additionally, increased S6 kinase activity leads to a reduction 467 in proliferating GNP due to premature cell cycle exit (61). 
To better understand how global mTOR overactivation impacts embryonic and early postnatal cerebellar development, we used $\mathrm{Tscl}^{+/-}$mice, often used as a mouse model to study mTORopathy-associated ASD. Because the mTOR kinase is an important regulator of translation (1), we first evaluated expression levels of mTOR pathway-related and cerebellar cell-specific genes in the developing cerebellum. The overall loss of correlation in distinct gene pairs we found in $\mathrm{Tscl}^{+/}$mice supports a deficient stability of the TSC1-TSC2 complex, as well as a dysregulation of translational machinery. This is in line with previous work demonstrating that increased mTOR function leads to an altered profile in neuronal genetic transcription (62). Thus, $T s c 1^{+/-}$haploinsufficiency alters the translational landscape of the cerebellum early in development, through the dysregulation of central mTOR-sensitive genes. To identify which cell types could potentially be more susceptible to mTOR overactivation, we analysed the relative expression of cerebellar-specific cell markers in the developing cerebellum. We found cerebellar lineage deficits as early as E18, which were further evident in the first week of postnatal development. Specifically, we found that cerebellar interneuronal precursors, characterized by the expression of $\operatorname{Pax} 2$, seemed to be particularly sensitive to global haploinsufficiency of $T s c 1$ and the consequent mTOR pathway overactivation. We observed that these interneuron precursors presented with hyperactive mTOR pathway. Additionally, this overactivation appeared to differentially affect the development of molecular and granular layer interneurons. The observed changes in Pax2 487 mRNA expression indicate that Tscl haploinsufficiency leads to a delay in the initiation of 488 Pax2 expression in embryonic development, causing its increased expression during later 489 postnatal periods. Thus, it is possible that disruption of cerebellar mTOR signalling primarily affects the maturation of interneurons rather than progenitor cell pool maintenance. 491 Alternatively, the deficient down-regulation of mTOR signalling found in P7 Tsc1 ${ }^{+/}$mice 492 could also contribute to an increase in cell proliferation, leading to elevated overall numbers of 
Pax $2^{+}$cells. These are pertinent hypotheses, as recent work in Drosophila has demonstrated

494 direct Pax2 expression modulation by the mTOR pathway (63). In Drosophila, D-Pax2 is a with Unkempt (Unk), a highly conserved zinc finger/RING domain protein, which is also highly expressed in mouse cerebellum (64). In Drosophila, Unk expression is negatively regulated by the mTOR pathway and $T s c 1$ mutant flies present with increased D-Pax2 expression (63). maturation, as evidenced by decreased PV staining in the cerebellum of $T s c 1^{+/-} \mathrm{P} 7$ mice. PV is

502 a calcium binding protein, abundant in Purkinje cells and cerebellar molecular layer interneurons (65). Thus, we focused on this population as they make up the majority of cerebellar interneuronal cells. During brain development, the initiation of PV expression coincides with the expression of a number of synaptogenesis markers, such as solute carrier family 32 and GABAA receptor a1 subunit (44). Furthermore, the amount of PV expression was shown to determine presynaptic calcium dynamics in cerebellar interneurons, modulating neurotransmitter release (44). Thus, the changes in PV expression levels and timing of the interneuronal maturation that we found in $T s c 1^{+/-}$mice, could potentially lead to deficits in synaptic integration in the cerebellum (66).

Behaviourally, Tsc1 mice models present with ASD-like features, including decreased

512 social interaction, increased repetitive behaviours and deficient reversal learning $(29,31)$. This

513 is a similar phenotype to the one found in PV knockout mice (67). Conversely, decreased numbers of PV positive cells are found in other models of ASD, namely Cntnap ${ }^{-/-}$, Shank $1^{-{ }^{-} \text {, }}$

515 Shank3B $^{-{ }^{-}}$, and Brinp3 $3^{--}(68-71)$. Based on this evidence, a recent review by Filice and 516 colleagues proposed the "Parvalbumin Hypothesis of Autism Spectrum Disorder", in which 517 down-regulation of parvalbumin expression leads to altered neuronal function and abnormal 
518 neurotransmitter release, in addition to increasing reactive oxygen species production and

519 dendritic branching (72). Thus, deficits in PV could be one of the mechanisms integrating

520 distinct high-risk mutations that lead to the development of ASD.

521

522

523

524

525

526

527

528

529

530

531

532

533

534

535

536

537

538

539

540

541

542 


\section{Competing interests}

544 The authors declare no competing interests.

545

\section{Acknowledgments}

547 We thank Ype Elgersma for the total S6 antibody; Chris de Zeeuw for the Pax2 and S6

548 antibodies; Roxanne ter Haar for the help in mouse genotyping and breeding; Elize Haasdijk

549 and Ivy Hau for their help with immunohistochemistry tissue processing. This research was

550 supported by the Netherlands Organization for Scientific Research and ZonMw

551 (VIDI/917.18.380,2018; AB).

552

553 Author contributions

554 IS, AS, and AB designed the study and analysis. IS, AS and MRO performed the qPCR and histological experiments. CO executed the western blot experiments. IS, AS, MRO and CO analysed the data. $\mathrm{CT}, \mathrm{MS}$ and $\mathrm{AB}$ supervised the project. IS, AS and $\mathrm{AB}$ wrote the first draft. All authors edited the manuscript.

\section{Data and code availability}

560 The raw data that support the findings of this study is available from the corresponding author upon request. The code is deposited at https://github.com/BaduraLab.

562 


\section{References}

569

1. Liu, G. Y. \& Sabatini, D. M. mTOR at the nexus of nutrition, growth, ageing and disease. Nat. Rev. Mol. Cell Biol. 21, 183-203 (2020).

2. Ka, M., Condorelli, G., Woodgett, J. R. \& Kim, W.-Y. mTOR regulates brain morphogenesis by mediating GSK3 signaling. Development 141, 4076-4086 (2014).

3. Easley, C. A., 4th et al. mTOR-mediated activation of $\mathrm{p} 70 \mathrm{~S} 6 \mathrm{~K}$ induces differentiation of pluripotent human embryonic stem cells. Cell. Reprogram. 12, 263-273 (2010).

4. Hartman, N. W. et al. mTORC1 targets the translational repressor 4E-BP2, but not S6 kinase 1/2, to regulate neural stem cell self-renewal in vivo. Cell Rep. 5, 433-444 (2013).

5. Moloney, P. B., Cavalleri, G. L. \& Delanty, N. Epilepsy in the mTORopathies: opportunities for precision medicine. Brain Commun 3, fcab222 (2021).

6. Nguyen, L. H. \& Bordey, A. Corrigendum: Convergent and Divergent Mechanisms of Epileptogenesis in mTORopathies. Front. Neuroanat. 15, 715363 (2021).

7. Haines, J. L. et al. Localization of one gene for tuberous sclerosis within 9q32-9q34, and further evidence for heterogeneity. Am. J. Hum. Genet. 49, 764-772 (1991).

8. Northrup, H. et al. Evidence for genetic heterogeneity in tuberous sclerosis: one locus on chromosome 9 and at least one locus elsewhere. Am. J. Hum. Genet. 51, 709-720 (1992).

9. Qin, J. et al. Structural Basis of the Interaction between Tuberous Sclerosis Complex 1 (TSC1) and Tre2-Bub2-Cdc16 Domain Family Member 7 (TBC1D7). J. Biol. Chem. 291, 8591-8601 (2016).

10. Crino, P. B. Evolving neurobiology of tuberous sclerosis complex. Acta Neuropathol. 125, 317-332 (2013).

11. Sarbassov, D. D. et al. Rictor, a novel binding partner of mTOR, defines a rapamycininsensitive and raptor-independent pathway that regulates the cytoskeleton. Curr. Biol. 14, 1296-1302 (2004). 
12. Kim, D.-H. et al. mTOR interacts with raptor to form a nutrient-sensitive complex that signals to the cell growth machinery. Cell 110, 163-175 (2002).

13. Masri, J. et al. mTORC2 activity is elevated in gliomas and promotes growth and cell motility via overexpression of rictor. Cancer Res. 67, 11712-11720 (2007).

14. Hoxhaj, G. et al. The mTORC1 Signaling Network Senses Changes in Cellular Purine Nucleotide Levels. Cell Rep. 21, 1331-1346 (2017).

15. McCabe, M. P. et al. Genetic inactivation of mTORC1 or mTORC2 in neurons reveals distinct functions in glutamatergic synaptic transmission. Elife 9, (2020).

16. McDonald, N. M. et al. Early autism symptoms in infants with tuberous sclerosis complex. Autism Res. 10, 1981-1990 (2017).

17. Kingswood, J. C. et al. TuberOus SClerosis registry to increase disease Awareness (TOSCA) - baseline data on 2093 patients. Orphanet J. Rare Dis. 12, 2 (2017).

18. American Psychiatric Association. Diagnostic and Statistical Manual of Mental Disorders: DSM-5TM. (American Psychiatric Association, 1980).

19. Elsabbagh, M. et al. Global prevalence of autism and other pervasive developmental disorders. Autism Res. 5, 160-179 (2012).

20. Hsu, L. C.-L. et al. Lhx2 regulates the timing of $\beta$-catenin-dependent cortical neurogenesis. Proc. Natl. Acad. Sci. U. S. A. 112, 12199-12204 (2015).

21. La Fata, G. et al. FMRP regulates multipolar to bipolar transition affecting neuronal migration and cortical circuitry. Nat. Neurosci. 17, 1693-1700 (2014).

22. Selby, L., Zhang, C. \& Sun, Q.-Q. Major defects in neocortical GABAergic inhibitory circuits in mice lacking the fragile X mental retardation protein. Neurosci. Lett. 412, 227232 (2007).

23. Wang, S. S.-H., Kloth, A. D. \& Badura, A. The cerebellum, sensitive periods, and autism. Neuron 83, 518-532 (2014). 
618

619

620

621

622

623

624

625

626

627

628

629

630

631

632

633

634

635

636

637

638

639

640

641

642

24. Limperopoulos, C. et al. Does cerebellar injury in premature infants contribute to the high prevalence of long-term cognitive, learning, and behavioral disability in survivors? Pediatrics 120, 584-593 (2007).

25. van der Heijden, M. E., Gill, J. S. \& Sillitoe, R. V. Abnormal Cerebellar Development in Autism Spectrum Disorders. Dev. Neurosci. 43, 181-190 (2021).

26. Kelly, E. et al. Regulation of autism-relevant behaviors by cerebellar-prefrontal cortical circuits. Nat. Neurosci. 23, 1102-1110 (2020).

27. Stoodley, C. J. et al. Altered cerebellar connectivity in autism and cerebellar-mediated rescue of autism-related behaviors in mice. Nat. Neurosci. 20, 1744-1751 (2017).

28. Badura, A. et al. Normal cognitive and social development require posterior cerebellar activity. Elife 7, (2018).

29. Tsai, P. T. et al. Autistic-like behaviour and cerebellar dysfunction in Purkinje cell Tsc1 mutant mice. Nature 488, 647-651 (2012).

30. Tsai, P. T. et al. Sensitive Periods for Cerebellar-Mediated Autistic-like Behaviors. Cell Rep. 25, 357-367.e4 (2018).

31. Goorden, S. M. I., van Woerden, G. M., van der Weerd, L., Cheadle, J. P. \& Elgersma, Y. Cognitive deficits in Tsc1+/- mice in the absence of cerebral lesions and seizures. Ann. Neurol. 62, 648-655 (2007).

32. Menashe, I., Grange, P., Larsen, E. C., Banerjee-Basu, S. \& Mitra, P. P. Co-expression profiling of autism genes in the mouse brain. PLoS Comput. Biol. 9, e1003128 (2013).

33. Li, Y. et al. Regionally specific TSC1 and TSC2 gene expression in tuberous sclerosis complex. Sci. Rep. 8, 13373 (2018).

34. Kwiatkowski, D. J. et al. A mouse model of TSC1 reveals sex-dependent lethality from liver hemangiomas, and up-regulation of p70S6 kinase activity in Tsc1 null cells. Hum. Mol. Genet. 11, 525-534 (2002). 
35. Carter, R. A. et al. A Single-Cell Transcriptional Atlas of the Developing Murine Cerebellum. Curr. Biol. 28, 2910-2920.e2 (2018).

36. Xu, D. et al. Identifying suitable reference genes for developing and injured mouse CNS tissues. Dev. Neurobiol. 78, 39-50 (2018).

37. Cheung, T. T., Weston, M. K. \& Wilson, M. J. Selection and evaluation of reference genes for analysis of mouse (Mus musculus) sex-dimorphic brain development. PeerJ 5, e2909 (2017).

38. Shaydurov, V. A., Kasianov, A. \& Bolshakov, A. P. Analysis of Housekeeping Genes for Accurate Normalization of qPCR Data During Early Postnatal Brain Development. $J$. Mol. Neurosci. 64, 431-439 (2018).

39. Rio, D. C., Ares, M., Jr, Hannon, G. J. \& Nilsen, T. W. Purification of RNA using TRIzol (TRI reagent). Cold Spring Harb. Protoc. 2010, db.prot5439 (2010).

40. Taylor, S. C. et al. The Ultimate qPCR Experiment: Producing Publication Quality,

41. Schindelin, J. et al. Fiji: an open-source platform for biological-image analysis. Nat. Methods 9, 676-682 (2012).

42. Wu, Y., Janmey, P. \& Sun, S. X. The Correlation Between Cell and Nucleus Size is Explained by an Eukaryotic Cell Growth Model. bioRxiv 2021.09.01.458491 (2021).

43. Haeri, M. \& Haeri, M. ImageJ plugin for analysis of porous scaffolds used in tissue doi:10.1101/2021.09.01.458491 engineering. J. Open Res. Softw. 3, (2015).

44. Simat, M., Ambrosetti, L., Lardi-Studler, B. \& Fritschy, J.-M. GABAergic synaptogenesis marks the onset of differentiation of basket and stellate cells in mouse cerebellum. Eur. J. Neurosci. 26, 2239-2256 (2007).

667 45. Maricich, S. M. \& Herrup, K. Pax-2 expression defines a subset of GABAergic 
interneurons and their precursors in the developing murine cerebellum. J. Neurobiol. 41, 281-294 (1999).

46. Lee, J. E., Lim, M. S., Park, J. H., Park, C. H. \& Koh, H. C. S6K Promotes Dopaminergic Neuronal Differentiation Through PI3K/Akt/mTOR-Dependent Signaling Pathways in Human Neural Stem Cells. Mol. Neurobiol. 53, 3771-3782 (2016).

47. Brown, A. M. et al. Molecular layer interneurons shape the spike activity of cerebellar Purkinje cells. Sci. Rep. 9, 1742 (2019).

48. Hoshino, M. Neuronal subtype specification in the cerebellum and dorsal hindbrain. Dev. Growth Differ. 54, 317-326 (2012).

49. Leto, K., Carletti, B., Williams, I. M., Magrassi, L. \& Rossi, F. Different types of cerebellar GABAergic interneurons originate from a common pool of multipotent progenitor cells. J. Neurosci. 26, 11682-11694 (2006).

680

50. Tavazoie, S. F., Alvarez, V. A., Ridenour, D. A., Kwiatkowski, D. J. \& Sabatini, B. L. Regulation of neuronal morphology and function by the tumor suppressors Tsc1 and Tsc2. Nat. Neurosci. 8, 1727-1734 (2005).

51. Glassmann, A. et al. Basic molecular fingerprinting of immature cerebellar cortical inhibitory interneurons and their precursors. Neuroscience 159, 69-82 (2009).

52. Zhang, L. \& Goldman, J. E. Generation of cerebellar interneurons from dividing progenitors in white matter. Neuron 16, 47-54 (1996).

53. Groteklaes, A., Bönisch, C., Eiberger, B., Christ, A. \& Schilling, K. Developmental Maturation of the Cerebellar White Matter-an Instructive Environment for Cerebellar Inhibitory Interneurons. Cerebellum 19, 286-308 (2020). morphologically identified interneurons in the vestibulocerebellum. J. Neurosci. 31, 712$724(2011)$. 
55. Yamanaka, H., Yanagawa, Y. \& Obata, K. Development of stellate and basket cells and their apoptosis in mouse cerebellar cortex. Neurosci. Res. 50, 13-22 (2004).

56. Grimaldi, P., Parras, C., Guillemot, F., Rossi, F. \& Wassef, M. Origins and control of the differentiation of inhibitory interneurons and glia in the cerebellum. Dev. Biol. 328, 422433 (2009).

57. Ka, M., Smith, A. L. \& Kim, W.-Y. MTOR controls genesis and autophagy of GABAergic interneurons during brain development. Autophagy 13, 1348-1363 (2017).

58. Angliker, N., Burri, M., Zaichuk, M., Fritschy, J.-M. \& Rüegg, M. A. mTORC1 and mTORC2 have largely distinct functions in Purkinje cells. Eur. J. Neurosci. 42, 2595$2612(2015)$.

59. Thomanetz, V. et al. Ablation of the mTORC2 component rictor in brain or Purkinje cells affects size and neuron morphology. J. Cell Biol. 201, 293-308 (2013).

705

60. Wu, C.-C. et al. mTORC1-Mediated Inhibition of 4EBP1 Is Essential for Hedgehog Signaling-Driven Translation and Medulloblastoma. Dev. Cell 43, 673-688.e5 (2017).

61. Mainwaring, L. A. \& Kenney, A. M. Divergent functions for eIF4E and S6 kinase by sonic hedgehog mitogenic signaling in the developing cerebellum. Oncogene 30, 1784$1797(2011)$.

62. Kim, J. K. et al. Brain somatic mutations in MTOR reveal translational dysregulations underlying intractable focal epilepsy. J. Clin. Invest. 129, 4207-4223 (2019).

63. Avet-Rochex, A. et al. Unkempt is negatively regulated by mTOR and uncouples neuronal differentiation from growth control. PLoS Genet. 10, e1004624 (2014).

64. Vinsland, E. et al. The zinc finger/RING domain protein Unkempt regulates cognitive flexibility. Sci. Rep. 11, 16299 (2021). terminals of cerebellar Purkinje cells and basket cells have higher levels of parvalbumin 
immunoreactivity than somata and dendrites: quantitative analysis by immunogold labeling. Exp. Brain Res. 93, 483-491 (1993).

720

721

66. Caillard, O. et al. Role of the calcium-binding protein parvalbumin in short-term synaptic plasticity. Proc. Natl. Acad. Sci. U. S. A. 97, 13372-13377 (2000).

67. Wöhr, M. et al. Lack of parvalbumin in mice leads to behavioral deficits relevant to all human autism core symptoms and related neural morphofunctional abnormalities. Transl. Psychiatry 5, e525 (2015).

68. Lauber, E., Filice, F. \& Schwaller, B. Dysregulation of Parvalbumin Expression in the Cntnap2-/- Mouse Model of Autism Spectrum Disorder. Front. Mol. Neurosci. 11, 262 (2018).

69. Filice, F., Vörckel, K. J., Sungur, A. Ö., Wöhr, M. \& Schwaller, B. Reduction in parvalbumin expression not loss of the parvalbumin-expressing GABA interneuron subpopulation in genetic parvalbumin and shank mouse models of autism. Mol. Brain 9, 10 (2016).

70. Schmitz-Abe, K. et al. Homozygous deletions implicate non-coding epigenetic marks in Autism spectrum disorder. Sci. Rep. 10, 14045 (2020).

71. Kobayashi, M., Hayashi, Y., Fujimoto, Y. \& Matsuoka, I. Decreased parvalbumin and somatostatin neurons in medial prefrontal cortex in BRINP1-KO mice. Neurosci. Lett. 683, 82-88 (2018).

72. Filice, F., Janickova, L., Henzi, T., Bilella, A. \& Schwaller, B. The Parvalbumin Hypothesis of Autism Spectrum Disorder. Front. Cell. Neurosci. 14, 577525 (2020). 